\title{
Growth of Oxide Nanorod, Nanotube and Nanocable Arrays through Template-based Sol Electrophoretic Deposition
}

\author{
H.M. Shang, Y. Wang, and Guozhong Cao* \\ Department of Materials Science and Engineering, University of Washington, Seattle, WA, USA
}

Keywords: Sol electrophoresis; template growth; nanorod, nanotube and nanocable arrays.

\begin{abstract}
This paper introduces a process for the growth of oxide nanorod, nanotube, and nanocable arrays that combines sol preparation and template-based electrophoretic deposition. Examples are shown that the sol electrophoretic deposition is an effective method for the formation of polycrystalline and single crystal oxide nanorod arrays, nanotube arrays and conformal coating of thin films of oxides on metal nanorods to produce metal-oxide core-shell nanocable arrays.
\end{abstract}

\section{Introduction}

Synthesis, characterization and application of nanowires, nanorods, nanotubes, nanocables, and nanobelts (also often referred to as one-dimensional nanostructure) comprise a significant aspect of today's endeavor in nanotechnology. In comparison with nanostructured materials in other forms, nanorod arrays offer several advantages for property study and practical applications. A significant progress has been made in the study on the physical properties of individual nanowires and nanorods by direct measuring the properties of individual nanostructures. However, such studies generally require dedicated preparation of experimental set-up and execution of measurements. For example, for the electrical conductivity measurement, patterned electrodes on a substrate are first created and, then, nanowires or nanorods are dispersed in an appropriate solvent or solution. Such prepared nanowire colloidal dispersion casts on the substrate with pattern electrodes. Measurements would be carried out after identifying individual nanowires or nanorods bridging two electrodes. There is very limited option for manipulation of nanowires or nanorods, and it is difficult to improve the contact between the sample and the electrodes to ensure a desired ohmic contact. For practical applications, the output or signals generated by single nanowire or nanorod based devices is small, signal to noise ratio is small, and thus highly sensitive instrumentation is required to accommodate such devices.

Evaporation-condensation growth, dissolution-condensation method, vapor-liquid-solid (VLS) growth method, template-based growth, and substrate ledge or step induced growth have been successfully demonstrated for the synthesis of various oxide nanowires and nanorods $[1,2,3,4,5]$. Template approach has been extensively investigated to prepare free-standing, non-oriented and oriented nanowires and nanorods. In addition to the desired pore or channel size, morphology, size distribution and density of pores, template materials must meet certain requirements. First, the template materials must be compatible with the processing conditions. Secondly, depositing materials or solution must wet the internal pore walls. Thirdly, for the synthesis of nanorods or nanowires, the deposition should start from the bottom or one end of the template channels and proceed from one side to another. Template-based growth often suffers from the polycrystalline nature of the resultant nanowires and nanorods, in addition to the difficulties to find appropriate templates with pore channels of desired diameter, length and surface chemistry and to remove the template completely without compromising the integrity of grown nanowires or nanorods.

\footnotetext{
*Corresponding author: gzcao@u.washington.edu
} 
The electrophoretic deposition technique has been widely explored, particularly for deposition of ceramic and organoceramic materials on cathode from colloidal dispersions $[6,7,8]$. When dispersed in a polar solvent or an electrolyte solution, the surface of nanoparticles develops an electrical charge via one or more of the following mechanisms: (1) preferential dissolution, (2) deposition of charges or charged species, (3) preferential reduction or oxidation, and (4) physical adsorption of charged species such as polymers. A combination of electrostatic forces, Brownian motion and osmotic forces would result in the formation of a so-called double layer structure. Upon application of an external electric field, charged particles are set in motion [9]. This type of motion is referred to as electrophoresis. When a charged particle moves, some of the solvent or solution surrounding the particle will also move with it, since part of the solvent or solution is tightly bound to the particle.

The mobility of a nanoparticle in a colloidal dispersion or a sol, $\mu$, is dependent on the dielectric constant of the liquid medium, $\varepsilon_{\mathrm{r}}$, the zeta potential of the nanoparticle, $\zeta$, and the viscosity, $\eta$, of the fluid. Several forms for this relationship have been proposed, such as the Hückel equation $[10,11,12,13]$ :

$$
\mu=\frac{2 \varepsilon_{r} \varepsilon_{0} \zeta}{3 \pi \eta}
$$

Electrophoretic deposition simply uses oriented motion of charged particles in an electrical field to grow films or monoliths by enriching the solid particles from a colloidal dispersion or a sol onto the surface of an electrode. If particles are positively charged (more precisely speaking, having a positive zeta potential), the deposition of solid particles will occur at the cathode. Otherwise, deposition will be at the anode. At the electrodes, the electrostatic double layers collapse and the particles coagulate, producing a porous materials made of compacted nanoparticles. Typical packing densities are far less than the 74 vol. \% theoretical density [14]. Many theories have been proposed to explain the processes at the deposition surface during electrophoretic deposition. However, the structural evolution on the growth surfaces is not well understood. Electrochemical process at the deposition surface or electrodes is complex and varies from system to system. The final density is dependent on the concentration of particles in sols or colloidal dispersions, zeta-potential, externally applied electric field, and reaction kinetics between particle surfaces. Slow reaction and slow arrival of nanoparticles onto the surface would allow sufficient particle relaxation on the deposition surface, so that a high packing density is expected.

\section{Experimental}

A combination of sol-gel processing and electrophoretic deposition has been used to synthesize a variety of oxide nanorods. Nanorod growth occurred on a working electrode of aluminum, with a $\mathrm{Pt}$ mesh counter electrode. The template membranes used for growth of the nanorods were track-etched hydrophilic polycarbonate (Millipore Isopore), with pore diameters of 50-200 nm, and a thickness of $10 \mu \mathrm{m}$, or $60 \mu \mathrm{m}$ thick anodic alumina (Whatman Anodisc), with $200 \mathrm{~nm}$ pores. The polycarbonate (PC) membrane and the working electrode are placed in a polypropylene filter holder, and held in place with a silicone gasket. This assembly is placed in contact with the sol. A Pt counter electrode is also placed in the sol, parallel to the working electrode. A slightly different arrangement was used with anodic alumina templates. Indium-tinoxide (ITO) glass was used as the working electrode and Pt mesh was used as the counter electrode. The alumina membrane contacting the conducting substrate is immersed in the sol parallel to the Pt counter electrode approximately $2.5 \mathrm{~cm}$ apart. The sol is initially drawn into the membrane pores by capillary action after membrane immersion into the sol. For electrophoretic growth, a potential of $5 \mathrm{~V}$ is applied between the electrodes, and held for up to 60 min for the polycarbonate membrane or up to $3 \mathrm{~h}$ for the alumina membrane. At the end of 
electrophoretic deposition, excess sol is blotted off the membrane. Samples prepared in this manner are dried at $\sim 100^{\circ} \mathrm{C}$ for several hours, then placed in an oven and fired for $60 \mathrm{~min}$ at $500^{\circ} \mathrm{C}$. This is to burn off the polycarbonate membranes, to make the nanorods dense, and to crystallize the sol in the desired phase. Additional wet chemical etching with $6 \mathrm{M} \mathrm{NaOH}$ is required to remove the alumina membrane since alumina withstands high temperatures, and epoxy adhesive is used to aid in the attachment of nanorods on ITO conducting substrates. Scanning electron microscopy (SEM, JEOL 840A) and transmission electron microscopy (TEM, JEOL 2010) were used to study the morphology and crystallinity of the nanorods. X-ray diffraction (XRD, Phillips PW1830) was used to determine the phases and crystal structures present, and to check for the presence of texture and degree of crystallinity.

\section{Results and discussion}

\subsection{Polycrystalline oxide nanorod arrays}

We succeeded in growth of various oxide nanorod arrays by combining sol-gel preparation with electrophoretic deposition $[15,16,17,18]$. The materials include anatase $\mathrm{TiO}_{2}$, amorphous $\mathrm{SiO}_{2}$, perovskite $\mathrm{BaTiO}_{3}$ and $\mathrm{Pb}(\mathrm{Ti}, \mathrm{Zr}) \mathrm{O}_{3}$, and layered perovskite $\mathrm{Sr}_{2} \mathrm{Nb}_{2} \mathrm{O}_{7}$. Figure 1 shows the SEM micrographs and XRD patterns of $\mathrm{TiO}_{2}$ nanorod arrays [18]. These nanorods have a uniform diameter throughout their entire length, with a surface that is smooth over much or all of the length. Comparing the various
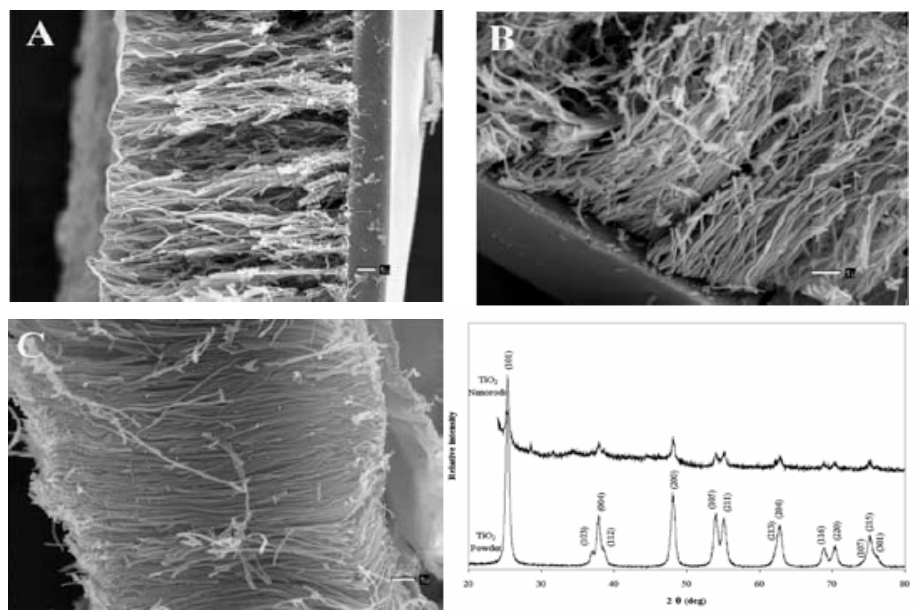

Fig. $1 \mathrm{SEM}$ micrographs of $\mathrm{TiO}_{2}$ nanorods grown by template-based electrochemically induced sol-gel deposition. rods, one can see that they all have roughly the same length and diameter. The image also shows that the rods arrange roughly parallel to one another over a broad area on the substrate. Figure 2 shows SEM micrographs of ITO. These samples show about $25-30 \%$ shrinkage in diameter compared to the original template diameter. The nanorods synthesized in this study are not straight, with a number of the nanorods exhibiting noticeable curvature.
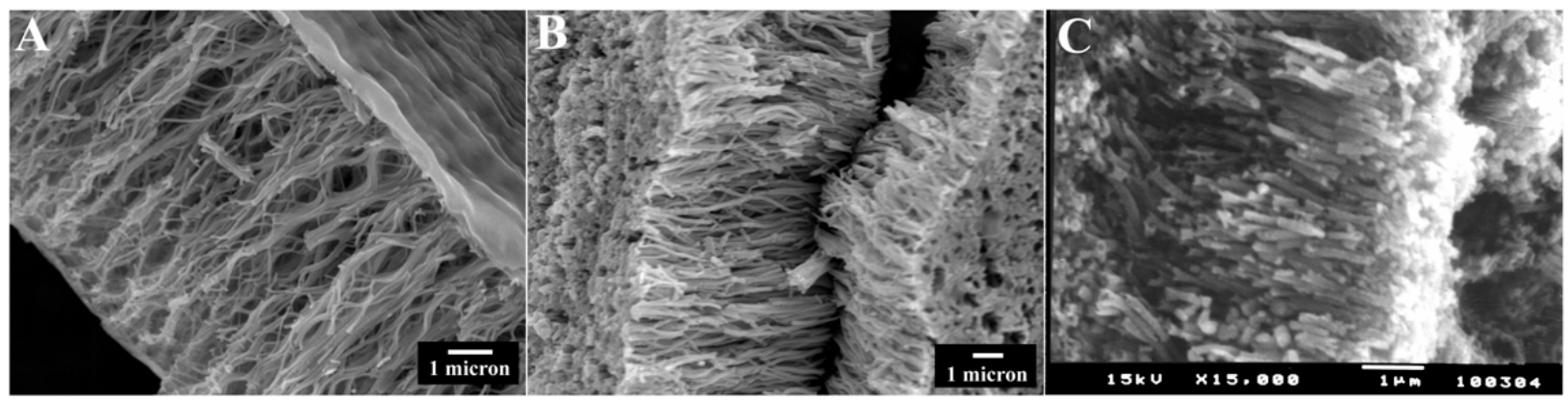

Fig. 2 Part A and B show SEM micrographs of nanorods of ITO grown in $100 \mathrm{~nm}$ (A) and $200 \mathrm{~nm}$ (B) templates at $1.33 \mathrm{~V} / \mathrm{cm}$ for $60 \mathrm{~min}$. Part $\mathrm{C}$ is an SEM micrograph of a sample where EPD was not used, showing poor quality nanorods. 


\subsection{Single crystal oxide nanorod arrays}

A modified version of sol electrophoretic deposition has been demonstrated to be capable of growing single crystalline titanium oxide and vanadium pentoxide nanorod arrays from respective $\mathrm{TiO}^{2+}$ and $\mathrm{VO}_{2}{ }^{+}$solutions. The formation of single crystal nanorods from solutions by $\mathrm{pH}$ change induced surface condensation has been proven by TEM analyses including high resolution image showing the lattice fringes and electron diffraction. The growth of single crystal nanorods by $\mathrm{pH}$ change induced surface condensation is attributed to evolution selection growth. Figure 3 shows SEM and typical TEM micrographs and selected-area electron diffraction pattern of $\mathrm{V}_{2} \mathrm{O}_{5}$ nanorods. It is well known that [010] or baxis is the fastest growth direction for $\mathrm{V}_{2} \mathrm{O}_{5}$ crystal $[19,20]$, which would explain why single crystal vanadia nanorods or a bundle of single crystal nanorods grow along the
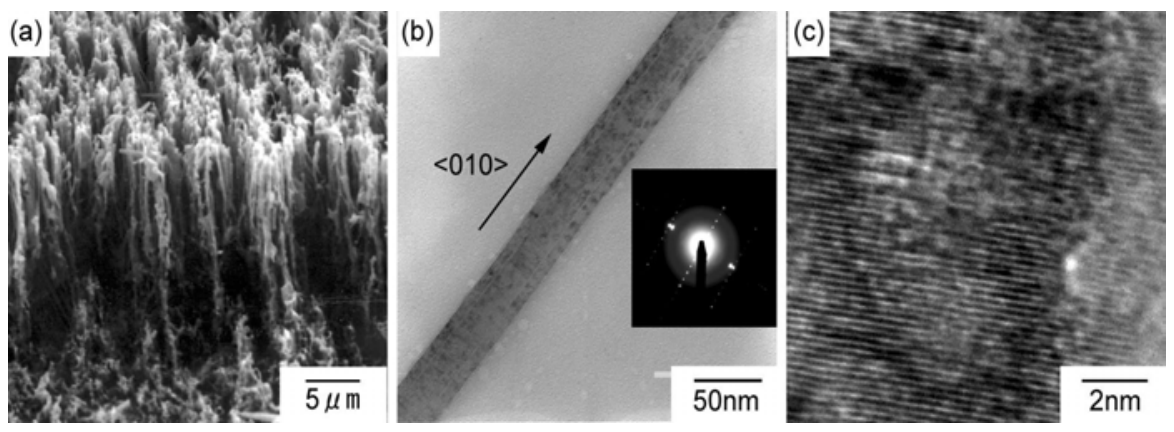

Fig. 3 SEM and TEM images of a $\mathrm{V}_{2} \mathrm{O}_{5}$ nanorod grown into a $200 \mathrm{~nm}$ membrane and its electron diffraction pattern. b-axis.

The growth of single crystal nanorods by $\mathrm{pH}$ change induced surface condensation is attributed to evolution selection growth, which is briefly summarized below. The initial heterogeneous nucleation or deposition on the substrate surface results in the formation of nuclei with random orientation. The subsequent growth of various facets of a nucleus is dependent on the surface energy and varies significantly from one facet to another. For one dimensional growth, such as film growth, only the highest growth rate with a direction perpendicular to the growth surface will be able to continue to grow. The nuclei with the fastest growth direction perpendicular to the growth surface will grow larger, whereas nuclei with slower growth rates will eventually cease to grow. Such a growth mechanism would result in the formation of columnar structured films with all of the grains having the same crystal orientation (known as textured films). In the case of nanorod growth inside a pore channel, such evolution selection growth is likely to lead to the formation of a single-crystal nanorod or a bundle of single-crystal nanorods per pore channel (Figure 4A).

Single crystal nanorods can also grown directly by conventional electrophoretic deposition. However, several requirements have to be met for such growth. First, the nanoclusters or particles in the sol must have a crystalline structure extended to the surface. Second, the deposition of nanoclusters on the growth must have a certain degree of reversibility so that the nanoclusters can rotate or reposition prior to its irreversible incorporation into the growth surface. Thirdly, the deposition rate must be slow enough to permit sufficient time for the nanoclusters to rotate or re-positioning. 
Lastly, the surface of nanoclusters must be free of strongly attached alien chemical species. Although precise control of all these parameters remains a challenge, the possibility of growth of single crystal nanorods through homoepitaxial aggregation of nanocrystals has been demonstrated $[21,22]$. The formation of single crystalline vanadium pentoxide nanorods by template-based sol electrophoretic deposition can be attributed to homoepitaxial aggregation of crystalline nanoparticles. Thermodynamically it is favorable for the crystalline nanoparticles to aggregate epitaxially; such growth behavior and mechanism have been well documented in literature $[23,24]$. In this growth mechanism, initial weak interaction between two nanoparticles allows rotation and migration relative to each other. Obviously, homoepitaxial aggregation is a competitive process and porous structure is expected to form through such homoepitaxial aggregation (as schematically illustrated in Figure 4B). Vanadium oxide particles in a typical sol are known to form ordered crystalline structure easily [25], so that it is reasonable to expect that homoepitaxial aggregation of vanadia nanocrystals from sol results in the formation of single crystal nanorods. Such formed single crystal nanorods are likely to undergo significant shrinkage when fired at high temperatures due to its original porous nature; $50 \%$ lateral shrinkage has been observed in vanadium pentoxide nanorods formed by this method. In addition, it might be possible that the electric field and the internal surface of pore channels both play a significant role in the orientation of nanorods, as suggested in literature [26,27].

\subsection{Nanotube and nanocable arrays}

Figure 5 shows SEM images of (a) top view and (b) side view of $\mathrm{V}_{2} \mathrm{O}_{5}$ nanotube array grown within the pores of PC membrane after the membrane is dissolved away in methylene chloride. These nanotubes stand apart from each other and project straight up from the substrate surface, with a length of $10 \mu \mathrm{m}$ (image not shown). As can be seen from the TEM images in Figure 5(c), the outer diameter of nanotube is about $200 \mathrm{~nm}$ and the inner diameter of nanotube is about $100 \mathrm{~nm}$. No electron diffraction pattern in TEM was observed, which suggests the amorphous nature of these nanotubes.

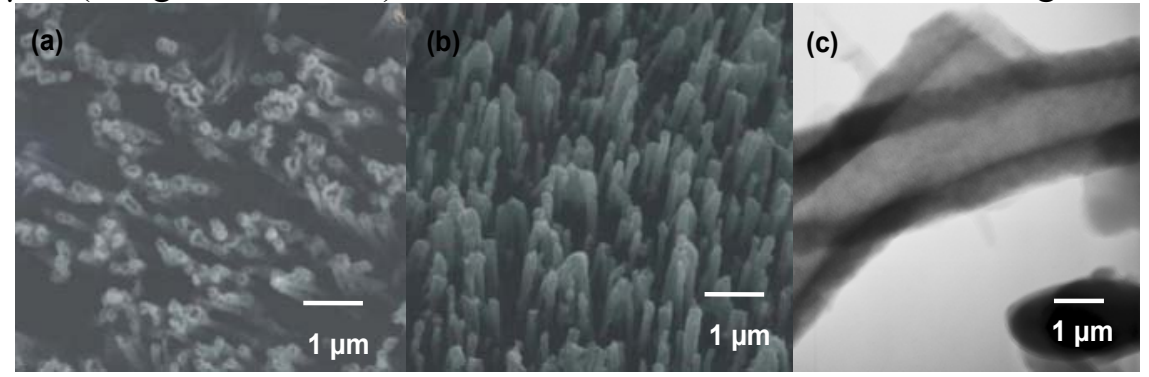

Fig. 5 SEM images of (a) top view and (b) side view of $\mathrm{V}_{2} \mathrm{O}_{5}$ nanotubes, and (c) TEM micrographs of isolated $\mathrm{V}_{2} \mathrm{O}_{5}$ nanotubes.

Figure 6 shows the SEM

micrographs of $\mathrm{Ni}$ nanorod arrays and $\mathrm{Ni}-\mathrm{V}_{2} \mathrm{O}_{5}$ core-shell nanocable arrays [28]. Ni nanorod arrays were grown in PC membrane by electrochemical deposition from nickel sulfate solution. After the removal of PC membrane by dissolving in ethylene chloride, vanadium pentoxide coating was deposited onto the surface of Ni nanorods by sol electrophoretic deposition. Figure 6(C) demonstrated that a thin vanadium pentoxide layer of approximately $30 \mathrm{~nm}$ in thickness was deposited on the surface of Ni nanorod with uniform confirmal coverage. Such electrical conductive core and dielectric shell nanocable structure can be made by a combination of electrochemical deposition and sol electrophoretic deposition.
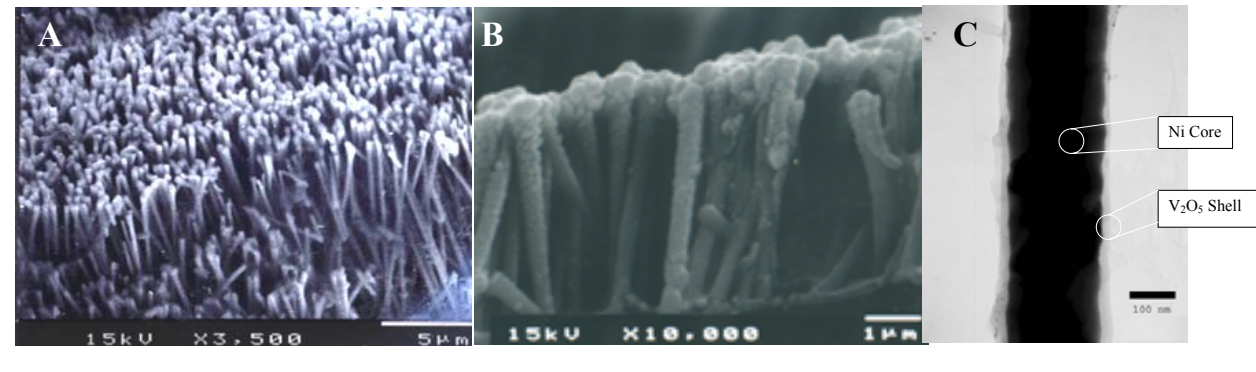

Fig. 6 SEM micrographs of Ni nanorod arrays (A) and $\mathrm{Ni}-\mathrm{V}_{2} \mathrm{O}_{5}$ core-shell nanocable arrays (B), and TEM micrograph of $\mathrm{Ni}-\mathrm{V}_{2} \mathrm{O}_{5}$ core-shell nanocable (C). 


\section{Summary}

Sol electrophoretic deposition is a simple combination of sol preparation and electrophoretic deposition. When combined with template-controlled growth, unidirectionally aligned and uniformly sized nanorod arrays over a large area can be readily formed. Although sol electrophoretic deposition primarily results in the formation of polycrystalline oxide nanorod arrays, it is demonstrated that single crystalline oxide nanorod arrays can be grown through either homoepitaxial aggregation or surface condensation induced by water hydrolysis. Nanotube and nanocable arrays can be readily grown with slightly modified deposition process.

Acknowledgements: HMS acknowledges Ford and JIN graduate fellowships, YW wants to acknowledge Ford, JIN and UIF graduate fellowships, and this work is supported in part by National Science Foundation (DMI-0455994).

\section{References}

[1] G.Z. Cao: Nanostructures and Nanomaterials: Synthesis, Properties and Applications (Imperial College, London 2004).

[2] Z.L. Wang: Nanowires and Nanobelts: Materials, Properties and Devices, Nanowires and Nonobelts of Functional Materials, Vol. 2. (Kluwer Academic Publishers, Boston 2003).

[3] Y. Xia, P. Yang, Y. Sun, Y. Wu, B. Mayers; B. Gates, Y. Yin, F. Kim, and H. Yan: Adv. Mater. Vol.15 (2003), pp.353.

[4] A. Huczko: Appl. Phys. A Vol. 70 (2000), pp.365.

[5] C. Burda, X. Chen, R. Narayanan, and M.A. El-Sayed: Chem. Rev. In press (2005).

[6] I. Zhitomirsky: Adv. Colloid Interf. Sci. Vol. 97 (2002), pp. 279.

[7] O.O. Van der Biest and L.J. Vandeperre: Annu. Rev. Mater. Sci. Vol. 29 (1999), pp. 327.

[8] P. Sarkar and P.S. Nicholson: J. Am. Ceram. Soc. Vol. 79 (1996), pp. 1987.

[9] A.C. Pierre: Introduction to Sol-Gel Processing (Kluwer, Norwell 1998).

[10] R.J. Hunter: Zeta Potential in Colloid Science: Principles and Applications (Academic Press, London 1981).

[11] C.J. Brinker and G. W. Scherer: Sol-Gel Science: the Physics and Chemistry of Sol-Gel Processing (Academic Press, San Diego 1990).

[12] J.D. Wright and N.A.J.M. Sommerdijk: Sol-Gel Materials: Chemistry and Applications (Gordon and Breach, Amsterdam 2001).

[13] D. H. Everett: Basic Principles of Colloid Science (the Royal Society of Chemistry, London 1988).

[14] W. D. Callister: Materials Science and Engineering: An Introduction (John Wiley \& Sons, New York 1997).

[15] S.J. Limmer, S. Seraji, M. J. Forbess, Y. Wu, T. P. Chou, C. Nguyen, and G.Z. Cao: Adv. Mater. Vol. 13 (2001), pp. 1269.

[16] S.J. Limmer, S. Seraji, M.J. Forbess, Y. Wu, T.P. Chou, C. Nguyen, and G.Z. Cao: Adv. Func. Mater. Vol. 12 (2002), pp. 59.

[17] S.J. Limmer and G.Z. Cao: Adv. Mater. Vol. 15 (2003), pp. 427.

[18] S.J. Limmer, T.P. Chou, and G.Z. Cao: J. Mater. Sci. Vol. 39 (2004), pp. 895.

[19] D. Pan, Z. Shuyuan, Y. Chen, and J. G. Hou: J. Mater. Res. Vol. 17 (2002), pp. 1981.

[20] V. Petkov, P. N. Trikalitis, E. S. Bozin, S. J. L. Billinge, T. Vogt, and M. G. Kanatzidis: J. Am. Chem. Soc. Vol. 124 (2002), pp. 10157.

[21] K. Takahashi, S.J. Limmer, Y. Wang, and G.Z. Cao: J. Phy. Chem. B Vol. 108 (2004), pp. 9795.

[22] G.Z. Cao: J. Phys. Chem. B Vol. 108 (2004), pp. 19921.

[23] R.L. Penn and J. F. Banfield: Geochim. Cosmochim. Acta Vol. 63(1999), pp. 1549.

[24] C. M. Chun, A. Navrotsky and I. A. Aksay: Proc. Microscopy and Microanalysis (1995), pp. 188.

[25] J. Livage: Coordination Chem. Rev. Vol. 178-180 (1998), pp. 999.

[26] K.V. Saban, J. Thomas, P.A. Varughese, and G. Varghese: Res. Technol. Vol. 37 (2002), pp. 1188.

[27] D. Grier, E. Ben-Jacob, R. Clarke, and L.M. Sander: Phys. Rev. Lett. Vol. 56 (1986), pp. 1264.

[28] K. Takahashi, Y. Wang and G.Z. Cao, J. Phys. Chem. B Vol. 109 (2005), pp.48. 